Technical Note

\title{
Investigation on mechanism of critical cavitating flow in liquid jet pumps under operating limits
}

\author{
X. Long ${ }^{\mathrm{a}, *}, \mathrm{H}$. Yao ${ }^{\mathrm{a}, \mathrm{b}}, \mathrm{J}$. Zhao ${ }^{\mathrm{c}}$ \\ a School of Power and Mechanical Engineering, Wuhan University, Wuhan 430072, China \\ ${ }^{\mathrm{b}}$ Nuclear Power Qinshan Joint Venture Company Limited, Haiyan 314300, China \\ ${ }^{\mathrm{c}}$ National Microgravity Laboratory, Institute of Mechanics, Chinese Academy of Sciences, Beijing 100190, China
}

\section{A R T I C L E I N F O}

\section{Article history:}

Received 9 May 2008

Received in revised form 24 November 2008

Available online 4 February 2009

\section{Keywords:}

Jet pump

Cavitation

Liquid-vapor two-phase flow

Critical flow

Operating limit

\begin{abstract}
A B S T R A C T
The critical cavitating flow in liquid jet pumps under operating limits is investigated in this paper. Measurements on the axial pressure distribution along the wall of jet pumps indicate that two-phase critical flow occurs in the throat pipe under operating limits. The entrained flow rate and the distribution of the wall pressure upstream lowest pressure section does not change when the outlet pressure is lower than a critical value. A liquid-vapor mixing shockwave is also observed under operating limits. The wave front moves back and forth in low frequency around the position of the lowest pressure. With the measured axial wall pressures, the Mach number of the two-phase cavitating flow is calculated. It's found that the maximum Mach number is very close to 1 under operating limits. Further analysis infers a cross-section where Mach number approaches to 1 near the wave front. Thus, the liquid-vapor mixture velocity should reach the local sound velocity and resulting in the occurrence of operating limits.
\end{abstract}

(c) 2008 Elsevier Ltd. All rights reserved.

\section{Introduction}

A jet pump is a kind of fluid machinery and mixing reaction equipment that transfers momentum from a high velocity primary jet flow to a secondary flow. A typical jet pump is composed of a nozzle, a suction chamber, a throat pipe and a diffuser as illustrated in Fig. 1. As high-pressure primary jet flow rushes out of the nozzle, it entrains the secondary flow from the suction chamber. Both flows exchange their momentum, energy and mass by turbulent mixing effect in the throat pipe. The mixed flow then gains more pressure energy when passing through the diffuser and discharges out. The absence of moving parts in a jet pump results in many advantages over other kind of pumps, such as simple structure, easy to machine, low capital cost, and convenience of operation and maintenance. The jet pump also has high reliability and adaptability for installation in remote or inaccessible locations to deal with poisonous, explosive, flammable or radioactive substance.

In this paper, both the primary and entrained fluids are liquid. So, for simplicity, the liquid jet pump is shortened as jet pump. As other kinds of pumps handling liquid, jet pumps are also hampered by cavitation. The intensive cavitation will bring about strong noise and vibration. The further development of cavitation will lead to the occurrence of the so-called operating limit which

\footnotetext{
* Corresponding author. Tel.:/fax: +86 2768772376 .

E-mail address: xplong@whu.edu.cn (X. Long).
}

the entrained flow rate reaches a peak and will not respond to the further decrease of outlet pressure. This results in the sharp drop-off in efficiency of jet pump and corresponding system, and sometimes endangers the safety of the whole system. Thus, as one of the major obstacles of its popularization, the existence of intensive cavitation and operating limit restrict the normal working range of jet pumps. It is also the bottleneck of the design of jet pumps. Therefore, this problem attracts many researchers' concerns, such as Cunningham et al. [4], Bonnington [3], and Lu and Shang [8], among many others.

Although almost the same description on the performance of the operating limit has been presented by every researcher, great differences exist on the interpretation of its mechanism. Also the cavitation process in jet pump was not well investigated. Kudirka and Decoster [6] suggested that the vapor bubble firstly occurs inside the jet boundary. With the decrease of the outlet pressure, the vapor bubbles occupy more space until they reach the wall of throat pipe. At this time the entrained flow will be choked and the flow rate will not increase with the decreases of outlet pressure. This explanation of mechanism of jet pump under operating limit is almost the same as those of Cunningham et al. [4] and Bonnington [3]. While, Lu and Shang [8] suggested that the change of the jet boundary layer is related to the speed ratio $M$. As $M$ increases, the jet boundary layer shrinks. When $M$ increases to a certain value, jet boundary layer will not increase but keep stable. Then, the entrained flow rate will not increase accordingly. 


\section{Nomenclatures}

\begin{tabular}{llll}
$A$ & cross-section area of pipe; & $W$ & mass flow rate; \\
$c$ & velocity of sound; & $z$ & coordinate along the main flow; \\
$C_{f}$ & frictional coefficient of two-phase flow; & \multicolumn{2}{l}{ Greek symbols } \\
$d$ & diameter of pipe; & $\alpha$ & volumetric void fraction; \\
$M$ & velocity ratio, $M=u_{o} / u_{s} ;$ & $\rho$ & density; \\
$M a$ & Mach number, $M a=u / c ;$ & \multicolumn{2}{l}{ area of the throat pipe } \\
$m$ & area ratio of jet pump, $m=\frac{\text { Subscripts }}{\text { area of the nozzle exit section; }}$ & $c$ & discharge flow of jet pump \\
$p$ & pressure; & $l$ & liquid phase \\
$Q$ & volumetric flow rate; & 0 & driving jet flow of jet pump \\
$q$ & flow rate ratio of jet pump, $q=Q_{s} / Q_{0} ;$ & $s$ & entrained flow of jet pump \\
$u$ & $u$ velocity; & $v$ & vapor phase
\end{tabular}

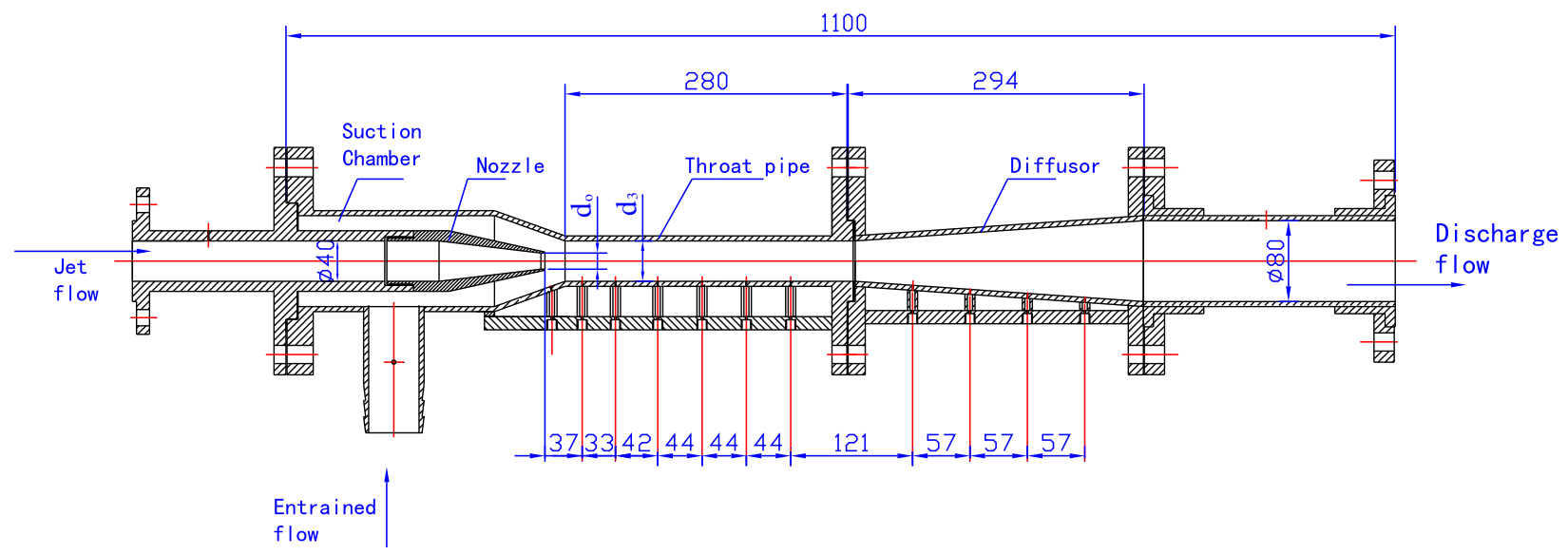

Fig. 1. Structure of jet pump (unit: $\mathrm{mm}$ ).

Wilson guessed that the operating limit might link with the sound speed of mixture of vapor and liquid [3]. Unfortunately, no further evidence was reported. Following this idea, Yao [10] developed an equation governing the cavitating flow in a horizontal cylindrical constant-area throat pipe under operating limit based on the hypotheses of iso-temperature process and homogenous vapor-liquid two-phase froth flow. This equation, marked as Eq. (1), shows that once the axial pressure gradient was known, the volumetric void fraction and the Mach number in the throat pipe can be obtained. It avoids the direct measurement of $M a$ and also offers an alternate method to obtain Ma. As a subsequent work, this paper will first make flow observation during different cavitation stage and then make measurement of pressure along throat pipe. The experimental data will be used to calculate $M a$ along the throat pipe. The results will be help to reveal the mechanism of operating limit.

$$
\left\{\begin{array}{l}
-\frac{d p}{d z}=2 C_{f} \frac{W^{2}}{A^{2} d} \frac{1}{\rho_{l}(1-\alpha)} /\left(1-M a^{2}\right) \\
\left(\frac{\alpha}{c_{v}^{2}} v_{v}+\frac{1-\alpha}{c_{l}^{2}} v_{l}\right)\left(\frac{1-\alpha}{v_{l}}+\frac{\alpha}{v_{v}}\right)^{-1} \frac{W^{2}}{A^{2}}=M a^{2}
\end{array}\right.
$$

\section{Experimental facility and procedure}

\subsection{Test rig and equipment}

The test rig was schematically illustrated in Fig. 2. The highpressure driving water $Q_{o}$ supplied by a centrifugal pump entrains the suction flow $Q_{s}$ from the water tank. The mixed stream $Q_{o}+Q_{s}$ discharges out to the reservoir. A plate is mounted to separate the water tank into two parts and also act as a spill wall to keep the water level of suction water constant. The flow-rate of primary flow and mixed flow are controlled by valves and measured by electromagnetic flow-meters. The water delivered to water tank is offered by another centrifugal pump via water supply pipe and valve. The driving water and entrained water are both from the same reservoir.

A pressure gauge is installed at the inlet of jet pump to monitor the driving pressure. Twelve pressure transducers are employed to measure the entrance pressure, outlet pressure and pressures along the throat pipe and the diffuser.

The replaceable nozzles are made of brass, while other parts of the jet pumps are made of organic glass. The diameter of the throat pipe is $40.01 \mathrm{~mm}$, while the outlet diameter of the nozzle is 16.00 and $17.93 \mathrm{~mm}$, respectively. So, the assembled jet pumps are of area ratios $m 6.25$ and 4.98, respectively. An organic glass quadrate box filling with water is installed around the throat pipe to reduce refraction and reflect for the purpose of observing clearly the cavitation phenomena with the help of a Nova-StrobeDA digital stroboscope.

The uncertainties of pressure transducers and electromagnetic flow-meters are $0.4 \%$. The maximum uncertainties of the pressure gradient and $\mathrm{Ma}$ are estimated no more than $1.5 \%$.

\subsection{Experimental procedure}

Before the test, the air in the system is expelled first by the self circulating. Then the driving pressure is adjusted to the preconcert- 




1, 7, 9, 11-valves; 2, 10-electromagnetic flowmeter; 3-air release valve; 4- pressure gauge; 5-jet pump;6- pressure transducer; 8-water tank.

Fig. 2. Sketch of the experimental rig.

ed value and the level of suction water is kept constant. By gradually adjusting the outlet valve, the cavitating flow can be clearly observed in different cavitation stages as shown in Fig. 3. With the opening of outlet valve, the mixed flow rate will increase. Once the mixed flow rate does not respond to the decrease of outlet pressure, strong noise and vibration can be felt, and the intensive cavitating flow can also be observed as shown in Fig. 3d. This is the socalled operation limit. During each cavitation stage, pressures and flow rates are collected by data acquisition system. Water temper- ature will be recorded after each test. Once completed, the driving pressure is changed, and the above procedures are repeated.

\section{Results and discussion}

\subsection{Flow observation under different cavitation stage}

Cavitation in a jet pump is confined coaxial jet cavitating flow with adverse pressure gradient, which is a kind of vortex
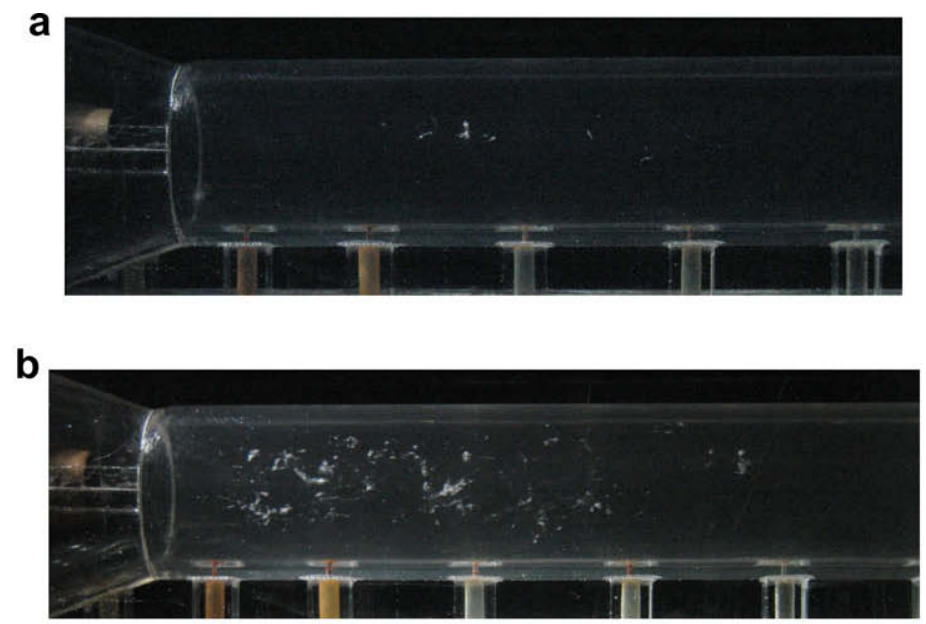

C

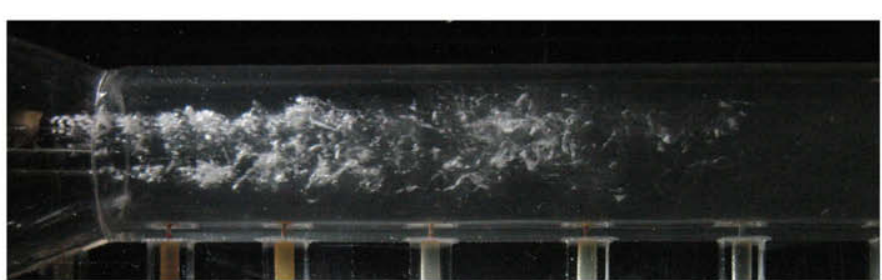

d

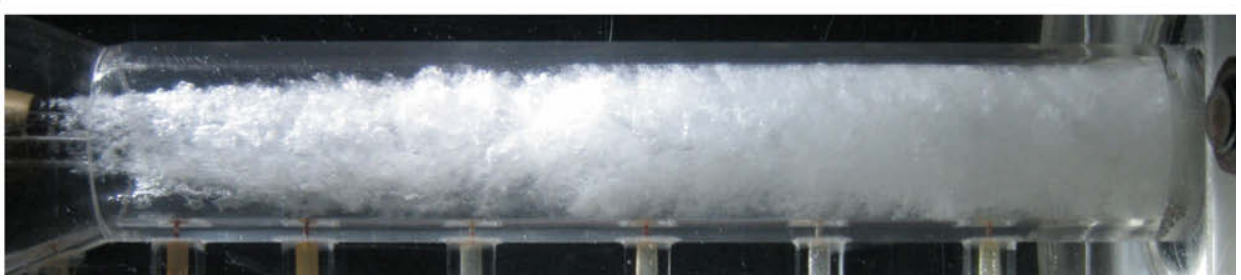

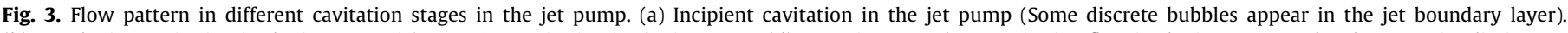
(b) Developing cavitation in the jet pump. (c) Intensive cavitation in the jet pump. (d) Intensive two-phase cavitating flow in the jet pump under the operating limit. 
cavitation. The experiment shows that cavitation in the jet pump might refers to incipient, developing and intensive cavitation under different operating conditions. As illustrated in Fig. 3a, vapor bubble will first appear at the jet boundary layer under a certain condition, and an incipient cavitation occurs. With the decrease of outlet pressure (or the increase of driving pressure), the number of bubbles increases (Fig. 3b), thus a developing cavitation is observed. The bubbles flow downstream with the main flow and collapse around the middle of the throat pipe. With further decrease of outlet pressure, more and more bubbles appear and congregate to cloud cavitation (Fig. 3c). This intensive and almost shape-fixed cloud was also observed by Winoto et al. [9] and Long and He [7]. With still further decrease of outlet pressure, the cavitation cloud extends to the end of the throat pipe and forms well mixed twophase bubbly flow (Fig. 3d). Strong noise and vibration can also be felt in this stage. The entrained flow rate reaches a peak and will not respond to the further decrease of outlet pressure. This means the jet pump operates in the operating limit.

Focusing on the flow pattern in the rear part of the throat pipe, some interesting results can be observed. Usually, it was believed that the bubbles will collapse in the diffuser because of pressure increase. But this is not always true. Sometimes, there exists an apparent interface in this section of the throat pipe (A-A in Fig. 4), across which the bubble density changes sharply. Upstream the interface the bubble density is high, while it becomes very low downstream the interface. This implies that there are many bubbles crumble at this interface. This is very similar to the shockwave of liquid-gas two-phase flow described by Brennen [1,2]. Also this is the major source of strong noise and vibration. The further observation shows that the interface is not stable but moves back and forth in the throat pipe as illustrated in Fig. 4a-c. Also the interface will sometimes extend to diffuser as illustrated in Fig. 4d. The preliminary test revealed that the frequency of the interface movement is the same as that of the induced low frequency noise.

\subsection{Wall pressure distribution along throat pipe and diffuser}

The wall pressure distribution along the throat pipe and the diffuser under different working status was measured. The pressures are fluctuation signals. The time-averaged pressures are shown in Fig. 5, where abscissa $Z$ represents the axial distance from the outlet of nozzle.

The curves marked " 1 " in Fig. 5 represent the wall pressure distribution under operating limits. The curves with higher outlet pressure than that of curve 1 are the wall pressure distributions under the so-called normal operating conditions. With the decrease of the outlet pressure, the pressure in throat pipe decreases gradually and the position of lowest pressure moves downstream gradually to the exit of the throat pipe. As the outlet pressure decreases to the value denoted by curve 1 , the operating limit occurs. At this time, the lowest pressure is close to but higher than the corresponding saturated vapor pressure.

From (Fig. 5), it's found that the negative pressure gradient increases until the pressure reaches the lowest value when the operating limit occurs. For a jet pump working under the operating limit, the lowest pressure occurs almost in the same position under different driving pressures. Because the size restriction of pressure transducer, pressures at more axial positions can not be obtained. So, this conclusion should be checked further in the future.

When liquid-gas two-phase flow in a pipe develops to critical flow, the velocity at the position of the lowest pressure reaches the local mixture sound velocity. From (Fig. 5), one can find that under operating limits, the pressure distribution upstream of the position of the lowest pressure will remain unchanged with the variation of outlet pressure. This phenomenon implies that the
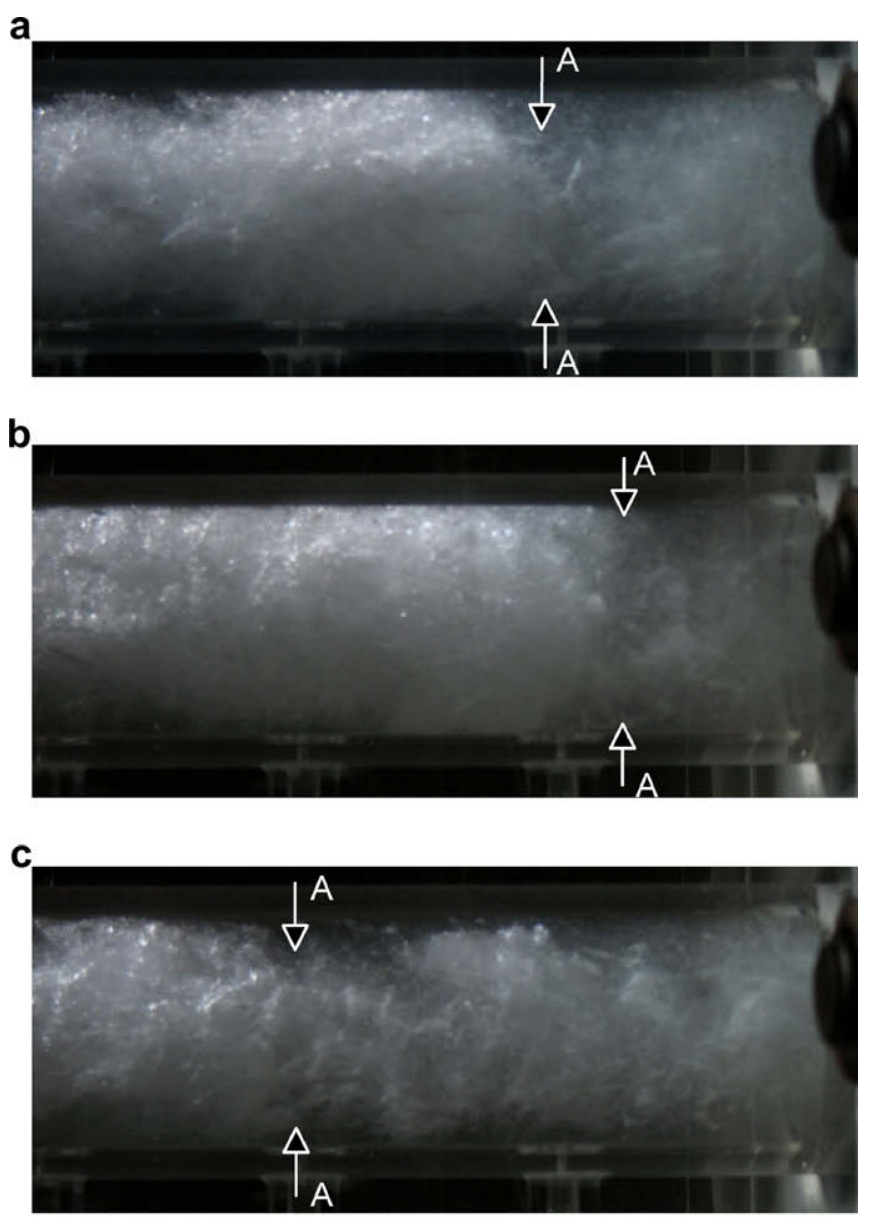

d

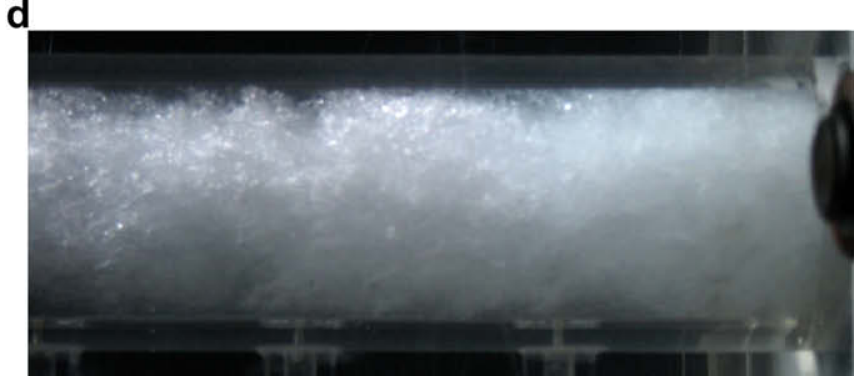

Fig. 4. The movement of interface. (a) Interface that bubble density changes sharply. (b) Interface moving forward to the diffuser. (c) Interface moving backward to the nozzle. (d) No interface appear in the throat pipe because it has moved into the diffuser.

froth flow within jet pumps under operating limits might develop to the two-phase critical flow.

\subsection{The mach number distribution under operating limits}

As stated before, once the relative operation parameters, especially the wall pressure distribution, have been obtained, the Mach number and its distribution can be obtained by solving Eq. (1). Table 1 is the calculated results of Ma distribution when operating limits occur, where $Z$ has the same meaning as that in Fig. 5.

For a jet pump working under the operating limit, $M a$ increases gradually at first. Near the end of throat pipe, it increases sharply, and then augments smoothly until reaching the peak value at the position of lowest pressure section. The maximum $M a$ is in the range of $0.929-0.942$ (Table 1 ), which is very close to 1 . This means that the velocity of the cavitating two-phase flow under the operating limits is very close to the corresponding sound velocity. 

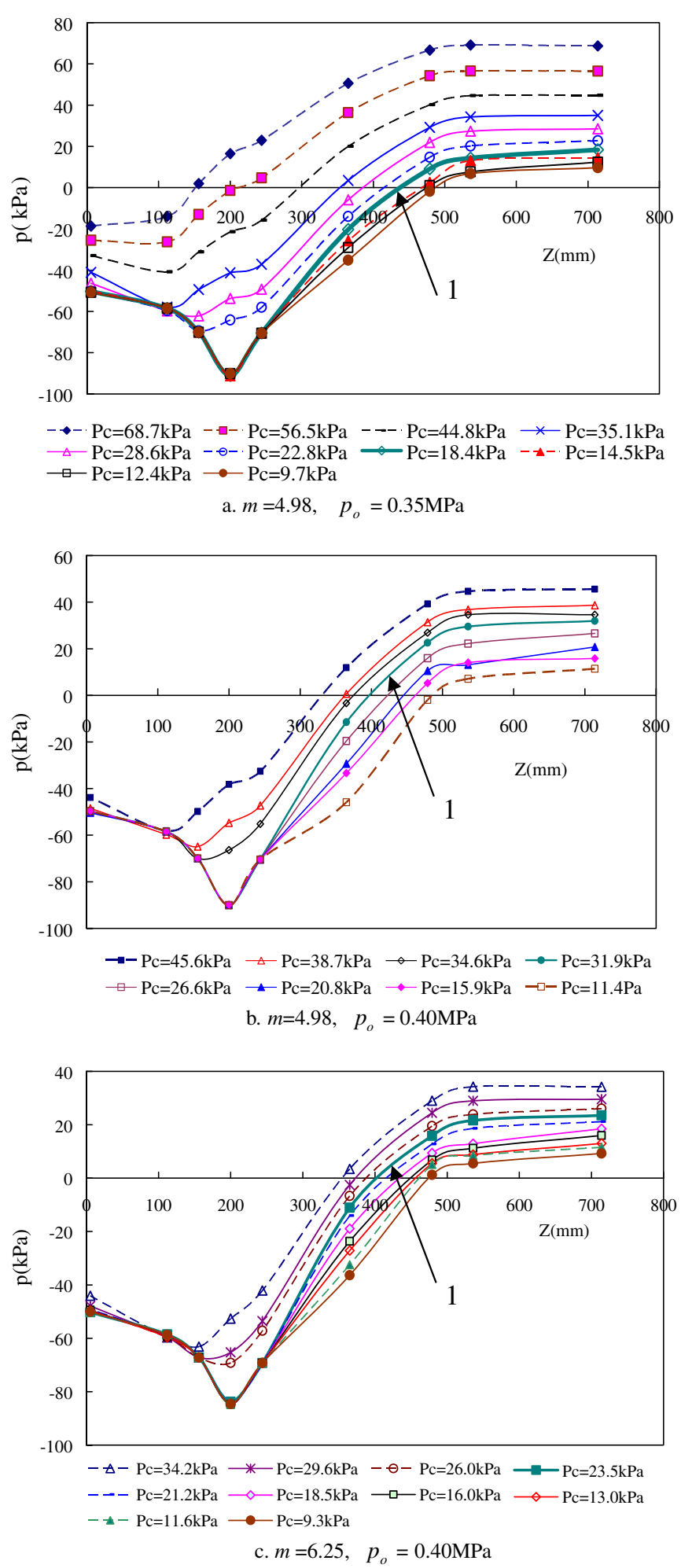

Fig. 5. Wall pressure distribution along throat pipe and diffuser.

Under post-limit operating conditions, the distribution of $\mathrm{Ma}$ along throat pipe has only a slighter change under different outlet pressure. This implies that $M a$ distribution is not sensitive to the variation of outlet pressure when operating limits occurs. The position of maximum $\mathrm{Ma}$ moves downstream with the increase of area ratio when the jet pumps work under the same driving pressure. This means that the increase of area ratio may delay the occurrence of operating limits.
Table 1

The distribution of $M a$ along the throat pipe under different discharge pressure.

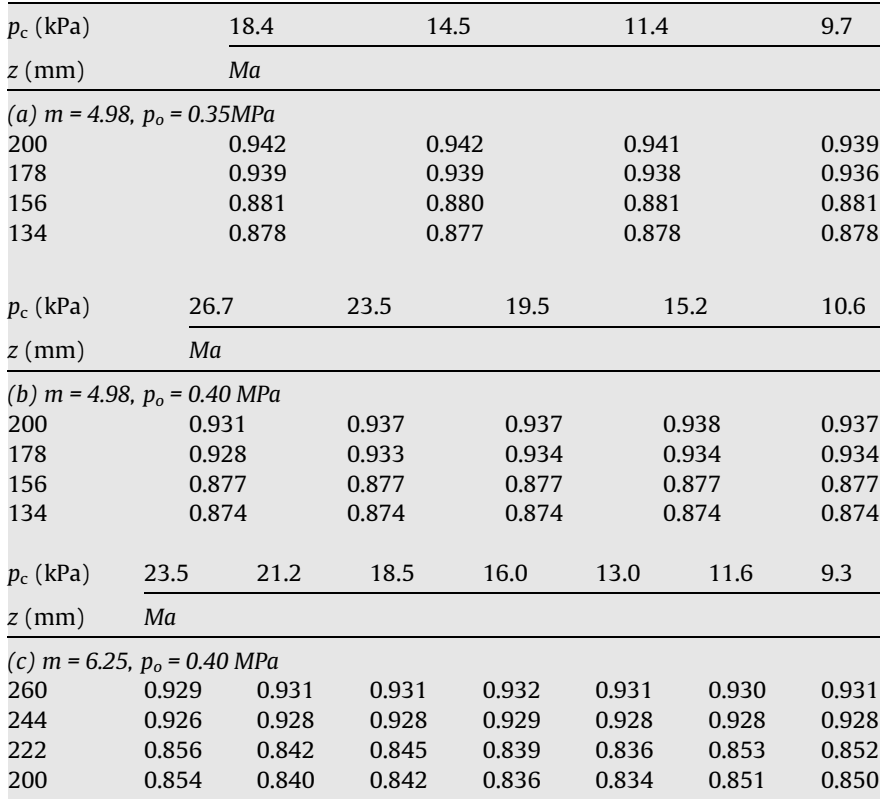

As observed, the shockwave interface or wave front moves back and forth in the rear section of the throat pipe. So, the position of lowest pressure as well as the biggest negative pressure gradient will also move as the wave front. The position of pressure transducers, however, is fixed. This means that the biggest negative pressure gradient might not be captured, resulting in that the calculated maximum Ma might not be the real one. If enough pressure transducers can be installed along the throat pipe, the biggest negative pressure gradient can be captured exactly, and then the exact maximum Ma can also be obtained. Nevertheless, from the above flow observation and $\mathrm{Ma}$ distribution, it can be concluded evidently that there should exist a cross-section near the position of lowest pressure in the rear part of throat pipe where Ma equals 1 and the critical two-phase flow occurs.

In the above calculations, the flow pattern in the rear part of the throat pipe was assumed to be homogenous froth flow. To validate the assumption, the void fraction $\alpha$ was also obtained by solving Eq. (1). It is in the range of 9-15\%. Comparing with the Baker Chart for horizontal two-phase flow patterns [5], the hypothesis of the froth flow is verified.

\section{Conclusion}

In the present paper, flow patterns in the throat pipe of liquid jet pumps under operating limit are observed, while the axial pressure distribution along the wall of jet pumps are also measured. Based on the analysis of the observations and the calculations of the distributions of the Mach number, it can be concluded that the critical liquid-vapor two-phase flow will occur when a jet pump works under the operating limit. In this situation, the velocity of mixed flow reaches the corresponding sound velocity. That's the reason why the outlet flow rate remains unchanged with the decrease of outlet pressure under a certain driving pressure when operating limits occurs.

\section{Acknowledgments}

The authors express their great thanks to National Natural Science Foundation of China (NSFC) for financial support under the grant of 50579060. The authors also express their thanks to Mr. Lei Wang and Mr. Zhiwei Guo for their help in the experiment. 


\section{References}

[1] C.E. Brennen, Cavitation and Bubble Dynamics, Oxford University Press, 1995

[2] C.E. Brennen, Fundamentals of Multiphase Flows, Cambridge University Press, 2005.

[3] S.T. Bonnington, Jet pumps and ejectors - a state of the art reviews and bibliographer, BHRA Fluid Eng. (1972).

[4] R.G. Cunningham, A.G. Hansen, T.Y. Na, Jet pump cavitation, Trans. ASME D 92 (1970) 483-494.

[5] S. Huang, B. Wei, Heat transfer of liquid vapor two phase flow, Press of Huazhong University of Science and Technology, 1988 (in Chinese).
[6] A.A. Kudirka, M.A. Decoster, Jet pump cavitation with ambient and high temperature water, ASME J. Fluids Eng. 101 (1979) 93-99.

[7] X. Long, P. He, Review on cavitation within jet pumps, Pump Technol. 4 (2003) 33-38 (in Chinese).

[8] H. Lu, H. Shang, Mechanism and calculation theory of jet pump cavitation, SCIENTIA SINICA A 11 (1987) 1174-1187.

[9] S.H. Winoto, D.A. Shah, ESE Te, Limiting flow condition in water jet pump, in: Proc. XIX IAHR Sym. on hydraulic machinery and cavitation, Singapore, vol.1, 1998, pp. 852-866.

[10] H. Yao, Study on critical behaviors of water jet pump, Master Dissertation, Wuhan University, Wuhan, China, 2006 (in Chinese). 